\title{
Comptonizing Efficiencies of IGR 17091-3624 and its similarity to GRS 1915+105
}

\author{
Partha Sarathi Pal ${ }^{1, *}$ \\ S. N. Bose National Centre For Basic Sciences, Kolkata. \\ Sandip K. Chakrabarti ${ }^{1}$
}

\begin{abstract}
Variability classes in the enigmatic black hole candidate GRS 1915+105 are known to be correlated with the variation of the Comptonizing Efficiency (CE) which is defined to be the ratio between the number of power-law (hard) photons and seed (soft) photons injected into the Compton cloud. Similarities of light curves of several variability classes of GRS 1915+105 and IGR 17091-3624, some of which are already reported in the literature, motivated us to compute CE for IGR 17091-3624 as well. We find that they are similar to what were reported earlier for GRS 1915+105, even though masses of these objects could be different. The reason is that the both the sizes of the sources of the seed photons and of the Comptonizing corona scale in the same way as the mass of the black hole. This indicates that characterization of variability classes based on CE is likely to be black hole mass independent, in general.
\end{abstract}

Keywords: Black Holes, Accretion disk, X-rays, Radiation mechanism.

\footnotetext{
*Corresponding author

Email addresses: parthasarathi.pal@gmail.com, partha.sarathi@boson.bose.res.in (Partha Sarathi Pal), chakraba@bose.res.in (Sandip K. Chakrabarti)

${ }^{1}$ S. N. Bose National Centre For Basic Sciences, Kolkata. JD Block, Salt Lake, Kolkata - 700098, India. Tel: +91-33-2335-5706/7/8 Extn: 374, Fax: +91-33-2335-9176.
} 


\section{Introduction}

It has been shown recently that the variability classes of GRS $1915+105$ could be characterized by Comptonizing Efficiency (CE) which is defined by the ratio of the power-law photons and injected seed photons averaged over the class duration (Pal et al. (2013) and references therein). Since the number of photons producing the power-law component is the same as the intercepted seed photons by the Comptonizing region, in reality, CE measures the geometry (more accurately, the optical depth) of the 'Compton cloud'. Not surprisingly, it was found that CE is very small for softer classes where the Compton cloud collapses as it cooled down and larger for harder classes where the Compton cloud is large. Earlier, for GRS 1915+105, these classes were characterized by conventional photon number ratios (so-called hardness ratios) in specific energy bands (Paul et al., 1997, 1998; Yadav et al., 1999; Muno et al., 1999; Belloni et al., 2000). Such ratios, and especially color-color diagrams drawn based on these ratios, appear to be different when a black hole of another mass is chosen since the definition of soft photon energy and hard photon energy depends on the mass. Indeed, one will have to fine tune energy bands suitably to obtain color-color diagrams of similar appearance for the same type of variability class. However, our motivation stems from the natural outcome of the two component advective flow (TCAF) paradigm (Chakrabarti \& Titarchuk (1995), hereafter CT95). Here, both the sources of soft photons (Keplerian disk) and Comptonized photons (Post-shock region of the advective flow component) are different regions of the TCAF and both of them scale with the mass of the black hole. Therefore our classification is expected to be mass independent. Thus CE, which is something similar to hardness ratio averaged over a short time scale compared to a class duration, can be used for defining variability classes. If $\mathrm{CE}$ remains similar for two objects having similar light curves, it also implies that they have similar spectral behavior and thus the classification is not superficial. In Pal et al. (2013), we prescribed a sequence in which all the variability classes of GRS $1915+105$ are expected to be manifested. Preliminary indications show (Pal et al., 2011) that indeed, observed variability class transitions follow our sequence. Our geometry based discussion presented above would therefore imply that for any other object, the sequence must also be the same. This makes our procedure to be a powerful tool not only to replace conventional hardness ratio plots by CE plots, but also has a predictability in terms of how the light curves and spectra are likely to appear in future. 
IGR J17091-3624 was first observed by an INTEGRAL Galactic Center Deep Exposure Coverage (Kuulkers et al., 2003). Later, the position was confirmed through Swift observation leaving two nearby blended candidates (Kennea \& Capitanio, 2007; Chaty et al., 2008). In early 2011, Swift-BAT detected an outburst from IGR J17091-3624 (Krimm et al., 2011). Optical/IR observations of the compact object was performed before and during the outburst (Torres et al., 2011). In this outburst, radio emission was detected which confirmed the presence of an accreting black hole candidate in its low-hard state (Corbel et al., 2011; Rodriguez et al., 2011). Rossi X-ray Timing Explorer (RXTE) detected low-frequency quasi-periodic oscillations (Rodriguez et al., 2011) and milli-Hertz and high frequency QPOs (Altamirano et al., 2011d; Altamirano \& Belloni, 2012). Some variability classes of the light curve that were seen in GRS 1915+105 were also observed for IGR 17091-3624 (Altamirano et al., 2011a,b,c; Pahari et al., 2012). As IGR J17091-3624 has a lower luminosity than GRS 1915+105 (Rodriguez et al., 2011), it is assumed that IGR J17091-3624 either consists of a very low mass black hole (Altamirano et al., 2011c) and/or it is located far away in the Galaxy with a distance of around $17-20 \mathrm{kpc}$ (Rodriguez et al., 2011; Altamirano et al., 2011c).

In the present paper, we show that IGR J17091-3624 has many apparently visually 'similar looking' variability classes (more than what is reported in the literature so far) as those of GRS 1915+105, though the time scales are different. Most surprisingly, we show that 'similar looking' variability classes of the two objects yield similar Comptonizing Efficiencies or CEs despite the fact that their masses could be totally different. Thus we can characterize each variability class by a unique $\mathrm{CE}$ value, independent of the mass of the black hole. Being similar CEs, the sequence in which hitherto unobserved class transitions are expected to take place for IGR J17091-3624 also becomes predictable. Since CE carries the information about geometry of the Compton cloud, we conclude that the Compton cloud geometry in IGR J17091-3624 also evolves in the same way as that in GRS 1915+105 (Pal et al., 2011, 2013; Pal \& Chakrabarti, 2014).

In the next Section, we present our analysis procedure and computation of Comptonizing Efficiency or CE for the IGR 17091-3624. We then present results of analysis of IGR 17091-3624. We compare CE values of IGR 170913624 with CE values of GRS 1915+105. Finally, we draw our conclusions. 
Table 1: Simultaneous Observation IDs along with common good time intervals of RXTEPCA and Swift-XRT data of IGR 17091-3624 analysed in this paper.

\begin{tabular}{cccc}
\hline \hline $\begin{array}{c}\text { Observation } \\
\text { date }\end{array}$ & $\begin{array}{c}\text { Swift-XRT } \\
\text { data id }\end{array}$ & $\begin{array}{c}\text { RXTE-PCA } \\
\text { data id }\end{array}$ & $\begin{array}{c}\text { Common GTI } \\
\text { sec }\end{array}$ \\
\hline $06 / 04 / 2011$ & 00031921040 & $96420-01-06-03$ & 461 \\
$10 / 05 / 2011$ & 00031921049 & $96420-01-11-03$ & 880 \\
$20 / 05 / 2011$ & 00031921053 & $96420-01-13-00$ & 1248 \\
$17 / 07 / 2011$ & 00035096020 & $96420-01-21-02$ & 411 \\
$29 / 07 / 2011$ & 00035096025 & $96420-01-23-00$ & 1057 \\
$31 / 07 / 2011$ & 00035096026 & $96420-01-23-02$ & 801 \\
$02 / 08 / 2011$ & 00035096027 & $96420-01-23-04$ & 454 \\
$04 / 08 / 2011$ & 00035096028 & $96420-01-23-06$ & 875 \\
$24 / 09 / 2011$ & 00035096044 & $96420-01-31-01$ & 2559 \\
$22 / 10 / 2011$ & 00035096057 & $96420-01-35-00$ & 552 \\
$26 / 10 / 2011$ & 00035096059 & $96420-01-35-03$ & 918 \\
\hline
\end{tabular}

\section{Analysis \& Calculation of CE}

In order to obtain spectrum in wider energy range, we have analysed data in the common good time intervals of RXTE-PCA and Swift-XRT in which IGR 17091-3624 was observed on days mentioned in Table. 1.

We plot 2.0-40.0 keV RXTE-PCA light curves of $1.0 \mathrm{sec}$ time bin of both IGR 17091-3624 and GRS 1915+105 in panels of Fig. 1. Several workers reported that IGR 17091-3624 sometimes shows variabilities similar to GRS 1915+105 (Altamirano et al., 2011a,b,c; Pahari et al., 2012). We show these and some more variability classes discovered by us in Fig. 1 by plotting them side by side for comparison. Some of these ( $\operatorname{such}$ as, $\beta$ ) may not look similar superficially as a whole. This is because duration of a dwell was not as large as should have been for IGR 17091-3624. If sufficient time was given to observe IGR 17091-3624, or time was scaled appropriately, the shapes would exhibit more resemblances.

\subsection{Spectral Analysis}

We analyze RXTE-PCA and Swift-XRT data given in Table. 1. We chose data sets by common MJDs of the outbursts observation, obtained from the NASA archive for both instruments. This allows us to study spectral 


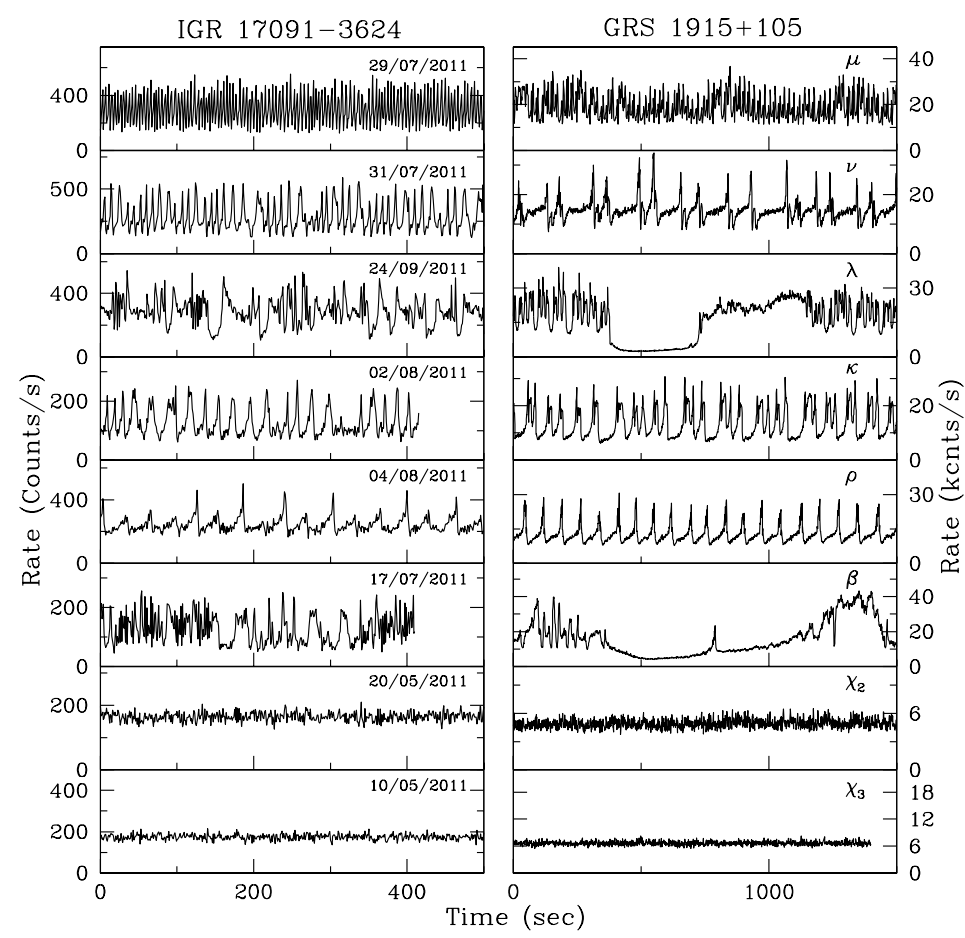

Figure 1: Comparison of the $2.0-40.0 \mathrm{keV} 1.0 \mathrm{sec}$ time bin RXTE-PCA light curves of IGR 17091-3624 (left) and GRS 1915+105 (right) to show their similarities. IGR 170913624 light curves clearly resemble with $\mu, \nu, \lambda, \kappa, \rho, \beta, \chi_{2}$ and $\chi_{3}$ classes of GRS 1915+105. 
properties in the wider range of energy. These data are downloaded from NASA Archive at HEASARC. RXTE and Swift data are reduced with HEASOFTv6.12 software.

For RXTE data reduction, we exclude data collected for elevation angles less than $10^{\circ}$, for offset greater than $0.02^{\circ}$ and those acquired during the South Atlantic Anomaly (SAA) passage. We selected RXTE PCU2 data as it was active during the time of observation. The RXTE-PCA spectra are extracted using "standard2" mode data which have 16 sec time resolution and energy selection from $3.0 \mathrm{keV}$ to $25 \mathrm{keV}$.

For Swift data reduction, the level 2 cleaned event files of SWIFT-XRT are obtained from window grade 0 events of timing (WT) mode data with xrtpipeline. The spectra are extracted from a $40 \times 10$ pixel region in the best source position. The background is estimated from an off-axis region of the same size. The ancillary response files (arfs) are extracted with xrtmkarf. The WT redistribution matrix file ( $\mathrm{rmf}$ ) version (v.12) is used in the spectral fits. The Swift-XRT spectra are extracted with 16 sec time resolution and energy selection from $0.5 \mathrm{keV}$ to $10 \mathrm{keV}$.

The combined $0.5 \mathrm{keV}$ to $25 \mathrm{keV}$ spectrum, consists of $0.5 \mathrm{keV}$ to $10 \mathrm{keV}$ Swift-XRT spectrum and $3.0 \mathrm{keV}$ to $25 \mathrm{keV}$ RXTE-PCA spectrum of the same good time interval and the same spectral resolution, are analysed using software package XSPEC 12.8.2. Here, each combined spectrum is averaged for 16 sec.

All spectra are fitted with diskbb and power-law model along with hydrogen column density for absorption due to interstellar medium $\mathrm{nH} 1.1 \times 10^{22} \mathrm{~cm}^{-2}$ (Krimm \& Kennea, 2011). We have multiplied a constant value along with the model components to compensate for the difference of normalizations between SWIFT-XRT and RXTE-PCA. During fitting of all spectra we used the technique introduced by Sobczak et al. (1999); Pal et al. (2011, 2013) to obtain spectral parameters to calculate number of seed photons and Comptonized photons (described below). We consider error-bars at $90 \%$ confidence level in each case under consideration.

\subsection{Calculation of Comptonizing Efficiency (CE)}

The fitting parameters are used to calculate the black body photons from the Keplerian disk and the power-law photons from the hot electron cloud. The number of black body photons are obtained from the fitted parameters of the multi-color disk black body model (Makishima et al., 1986). This is 
given by,

$$
f(E)=\frac{8 \pi}{3} r_{\text {in }}^{2} \cos i \int_{T_{\text {out }}}^{T_{\text {in }}}\left(T / T_{\text {in }}\right)^{-11 / 3} B(E, T) d T / T_{\text {in }}
$$

where, $B(E, T)=\frac{E^{3}}{(\exp E / T-1)}$ and $r_{i n}$ can be calculated from,

$$
K=\left(r_{i n} /(D / 10 k p c)\right)^{2} \cos i,
$$

where, $K$ is the normalization of the blackbody spectrum obtained after fitting, $r_{i n}$ is the inner radius of the accretion disk in $k m, T_{i n}$ is the temperature at $r_{i n}$ in $\mathrm{keV}, D$ is the distance of the compact object in kpc and $i$ is the inclination angle of the accretion disk. Here, both the energy and the temperature are in keV. The black body flux, $f(E)$ in photons $/ \mathrm{s} / \mathrm{keV}$ is integrated between $0.1 \mathrm{keV}$ to the maximum energy $d b b_{e} . d b b_{e}$ is the highest energy up to which the spectrum is fitted with diskbb model alone (Sobczak et al., 1999; Pal et al., 2011, 2013). This gives us $N_{B B}$, the rate at which black body photons are emitted.

The Comptonized photons $N_{P L}$ that are produced due to inverse-Comptonization of the soft black body photons by 'hot' electrons in the Compton cloud are calculated by fitting with the power-law given below,

$$
P(E)=N E^{-\alpha},
$$

where, $\alpha$ is the power-law index and $N$ is the total photons $/ \mathrm{s} / \mathrm{cm}^{2} / \mathrm{keV}$ at $1 \mathrm{keV}$. It is reported Titarchuk (1994) that the Comptonization spectrum will have a peak at around $3 \times T_{\text {in }}$. The power-law equation is integrated from $3 \times T_{\text {in }}$ to $40 \mathrm{keV}$ to obtain the rate of emitted Comptonized photons. We have chosen the upper energy limit for calculation of Comptonized photons to be $40 \mathrm{keV}$ as this is the upper energy limit of the PCA detector. We do not anticipate that our results would change significantly even when higher energy photons are included since the number of photons decreases rapidly with energy. In Table. 2 we give parameters of spectral analysis of XRT and PCA spectrum. Fig. 2 shows a fitted 16 sec sample of simultaneous SWIFTXRT and RXTE-PCA spectra for IGR 17091-3624 during 04/08/2011. The spectra are fitted with diskbb and power-law components. The photon numbers and $\mathrm{CE}$ are calculated from the fitting parameters. The diskbb spectrum is simulated with $T_{\text {in }}=1.31_{-0.07}^{+0.07} \mathrm{keV}$. The calculated number of blackbody photons from $0.1-6.0 \mathrm{keV}$ is $18.42_{-2.87}^{+3.46} \mathrm{kcnts} / \mathrm{s}$. Then spectra fitted with 


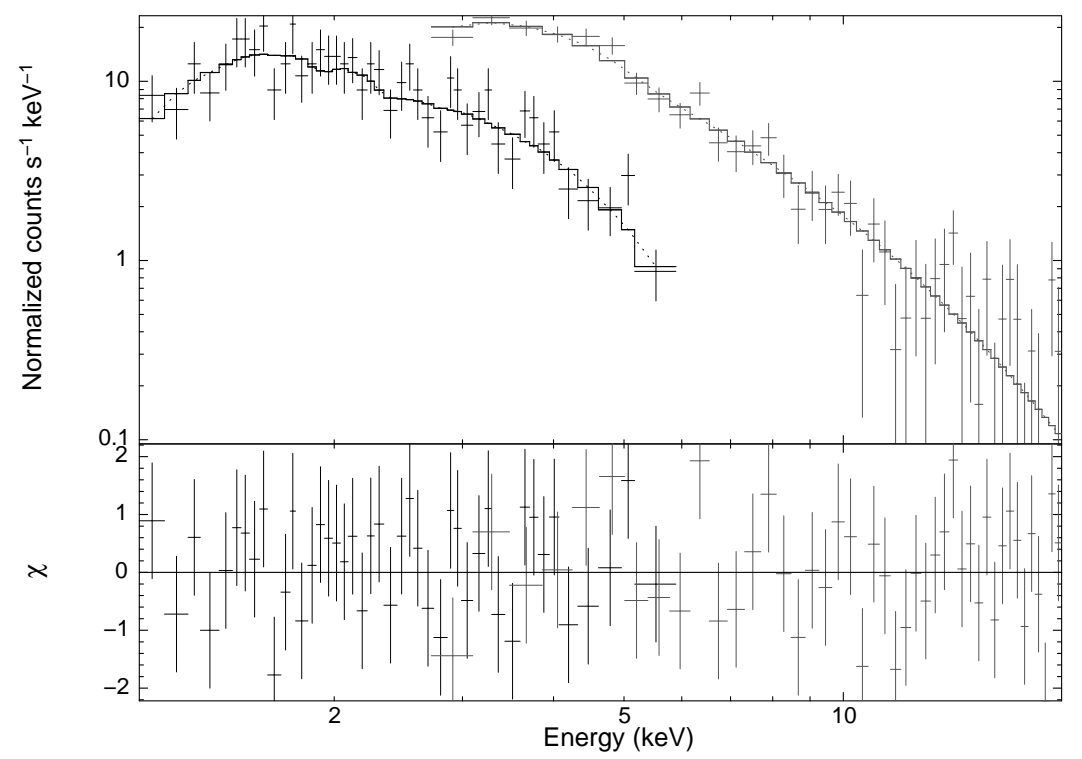

Figure 2: A simultaneous 16 sec sample of XRT and PCA fitted spectra during 04/08/2011. The black curves represents the SWIFT-XRT data and gray curves represent RXTE-PCA data. The spectra are fitted with diskbb and power-law components.

diskbb and power-law while diskbb components remained frozen to the values obtained from previous fitting of spectra with diskbb model only. The power-law spectrum is simulated with power-law index $=1.19_{-0.76}^{+0.39}$. The calculated number of Comptonized photons between $3.93-40.0 \mathrm{keV}$ is $0.04_{-0.006}^{+0.005}$ kcnts/s. This means that only $0.21_{-0.08}^{+0.07 \%}$ blackbody photons are actually Comptonized. For the intermediate states we have computed twice, one for burst-off (low count) state which is harder (denoted by 'h') and the other for the burst-on state (high count) which is softer (denoted by 's'). Details of these states are presented in Pal et al. (2013) and references therein.

At any given instant, the ratio $N_{P L} / N_{B B}$ is the Comptonizing Efficiency (CE). This is similar to a 'hardness ratio' though the energy ranges of the hard and the soft photons are automatically determined by the fitting process as described above. In TCAF paradigm (CT95), power-law photons originate through Comptonization of the post-shock region of the low-angular momentum advective component and seed photons are from the Keplerian component. However, sizes of both the components scale with the mass of the black hole and thus we expect $\mathrm{CE}$ to be mass independent. Thus using 
Table 2: Parameters for the spectral fits of simultaneous 16s bin of PCA and XRT spectra with diskbb plus power-law models for all observation dates. $h$ and $s$ are sample parameters for harder and softer states in a class with intermediate states. $T_{i n}$ is the black body temperature obtained from fitting. $d b b_{e}$ is the upper limit of the disk blackbody spectrum. ${\tilde{\chi_{\dagger}}}^{2}$ represents the reduced $\chi^{2}$ value of $0.9-d b b_{e} \mathrm{keV}$ spectra fitted with diskbb model only. Soft photon rate includes blackbody photons in the $0.1-d b b_{e} \mathrm{keV}$. 'power-law' is the power-law index $\alpha$ obtained from fitting. Hard photon rate includes Comptonized photons emitted in $3 \times T_{i n}-40 \mathrm{keV}$. CE is the Comptonized Efficiency. $\chi_{\ddagger}^{2}$ is reduced $\chi^{2}$ value of $0.9-25.0 \mathrm{keV}$. The sequence is according to the increased CE averaged over a variability class (same as in Fig. 5 below).

\begin{tabular}{|c|c|c|c|c|c|c|c|c|c|}
\hline \multicolumn{2}{|l|}{ Date } & $\begin{array}{c}T_{i n} \\
(\mathrm{keV})\end{array}$ & $\begin{array}{c}d b b_{e} \\
(\mathrm{keV})\end{array}$ & $\begin{array}{c}{\tilde{\chi_{\dagger}}}^{2} \\
(\mathrm{dofs})\end{array}$ & $\begin{array}{c}\text { Soft } \\
\text { Photon } \\
(\text { kphtns/s) }\end{array}$ & $\begin{array}{c}\text { Power law } \\
\text { index }\end{array}$ & $\begin{array}{c}\text { Hard } \\
\text { Photon } \\
(\mathrm{kphtns} / \mathrm{s})\end{array}$ & $\begin{array}{l}\mathrm{CE} \\
(\%)\end{array}$ & $\begin{array}{c}{\tilde{\chi_{\ddagger}}}^{2} \\
\text { (dofs) }\end{array}$ \\
\hline $9 /$ & - & $.66_{-0.08}^{+0.09}$ & 10 & $0.94(65)$ & $\begin{array}{l}16.89_{-2.47}^{+2.96} \\
\end{array}$ & $1.95_{-0.50}^{+0.52}$ & $0.2_{-0.01}^{+0.02}$ & $0.1_{-0.03}^{+0.02}$ & $.88(114)$ \\
\hline $26 / 10 / 2011$ & - & $.84_{-0}^{+0}$ & 10 & $.34(64)$ & $14.48_{-2.47}^{+3.09}$ & $2.50^{+}$ & $0.01_{-0}^{+0}$ & $0.07_{-0}^{+0}$ & $08(114)$ \\
\hline $22 / 10 / 2011$ & & $.97_{-0}^{+0}$ & 10 & $24(62)$ & $15.02_{-2.68}^{+3.41}$ & $2.15_{-0.40}^{+0.40}$ & $0.02_{-0}^{+0}$. & $0.12_{-0.02}^{+0.07}$ & $1.06(112)$ \\
\hline \multirow[t]{2}{*}{$31 / 07 / 2011$} & 11 & $.45_{-0}^{+0}$ & 10 & $1.09(65)$ & $19.45_{-2.89}^{+3.44}$ & $1.86_{-0.50}^{+0.50}$ & $0.02_{-0.004}^{+0.003}$ & $0.10_{-0.04}^{+0.03}$ & $.95(114)$ \\
\hline & $\mathrm{S}$ & $1.57_{-}^{+}$ & 10 & $0.86(57)$ & $16.19_{-2}^{+3}$ & 2.12 & $0.01_{-}^{+}$ & $.06_{-}^{+}$ & $0.78(106)$ \\
\hline 11 & 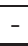 & $1.48_{-1}^{+1}$ & 10 & $.35(73)$ & $20.39_{-3.10}^{+3.73}$ & $2.07_{-0.72}^{+0.37}$ & $0.03_{-0}^{+0}$ & $0.12_{-1}^{+1}$ & $1.05(123)$ \\
\hline \multirow[t]{2}{*}{$02 / 08 / 2011$} & $\mathrm{n}$ & $1.53_{-0.1}^{+0.1}$ & 10 & $1.59(54)$ & $13.82_{-2.61}^{+3.34}$ & $1.61_{-0.31}^{+0.25}$ & $0.03_{-0}^{+0}$ & $0.23_{-0}^{+0}$ & $1.02(103)$ \\
\hline & $\mathrm{s}$ & $1.56_{-0.07}^{+0.08}$ & 10 & $1.28(71)$ & $19.20_{-2.75}^{+3.29}$ & $2.30_{-0.78}^{+0.46}$ & $0.02_{-0}^{+0}$ & $0.10_{-0}^{+0}$ & $1.06(120)$ \\
\hline \multirow[t]{2}{*}{$04 / 08 / 2011$} & $\mathrm{~b}$ & $1.31_{-}^{+}$ & 6.0 & 0) & $18.42_{-2}^{+3}$ & 1.19 & $0.04_{-}^{+}$ & $0.21_{-}^{+}$ & .97( \\
\hline & $\mathrm{S}$ & $1.53_{-0.09}^{+0.10}$ & 9.0 & (53) & $13.95_{-2.55}^{+3.20}$ & $2.00_{-0.02}^{+0.03}$ & $0.01_{-0}^{+0}$ & $0.07_{-0.06}^{+0.03}$ & $.95(102)$ \\
\hline \multirow[t]{2}{*}{$06 / 04 / 2011$} & $h$ & $1.16_{-0}^{+0}$ & 3.0 & $0.87(28)$ & $16.48_{-3.60}^{+4.73}$ & $1.49_{-0.85}^{+0.63}$ & $0.03_{-0}^{+0}$ & $0.20_{-0.13}^{+0.15}$ & $0.75(111)$ \\
\hline & $\mathrm{s}$ & $1.40_{-0.11}^{+0.20}$ & 6.0 & $.05(40)$ & $17.46_{-3.78}^{+4.04}$ & $2.17_{-0.75}^{+0.45}$ & $0.01_{-0}^{+0}$ & $0.09_{-0.05}^{+0.06}$ & $0.86(124)$ \\
\hline \multirow[t]{2}{*}{$17 / 07 / 2011$} & $n$ & $1.14_{-0}^{+0}$ & 6.0 & $1.66(23)$ & $84.59_{-19.90}^{+15.09}$ & $2.46_{-0.53}^{+0.78}$ & $0.27_{-1}^{+1}$ & $0.32_{-}^{+1}$ & $0.79(90)$ \\
\hline & $\mathrm{s}$ & $1.87_{-0}^{+0}$ & 10 & $0.79(90)$ & $61.30_{-16.55}^{+21.09}$ & $2.98_{-0.27}^{+0.32}$ & $0.09_{-0.026}^{+0.020}$ & $0.15_{-0.04}^{+0.04}$ & $0.82(82)$ \\
\hline $20 / 05 / 2011$ & - & $1.12_{-0.17}^{+0.21}$ & 6.5 & $1.3(37)$ & $13.73_{-3.54}^{+5.14}$ & $2.19_{-0.30}^{+0.24}$ & $0.08_{-0.01}^{+0.01}$ & $0.58_{-0.24}^{+0.29}$ & $0.95(95)$ \\
\hline $10 / 05 / 2011$ & - & $1.11_{-0.17}^{+0.26}$ & 3.1 & $1.0(21)$ & $7.52_{-2.96}^{+6.45}$ & $2.02_{-0.63}^{+0.33}$ & $0.06_{-0.02}^{+0.08}$ & $0.77_{-0.38}^{+0.37}$ & $0.93(96)$ \\
\hline
\end{tabular}


$\mathrm{CE}$ in place of conventional hardness ratio would would be more appropriate in classification of various light curves and variability types.

\section{Results}

On 06/04/2011, the PCA count rate varies from 50 to 200 counts/s. The light curve matches with a part of $\beta$ class of GRS 1915+105. Here, the value of CE varies from $0.08 \%$ to $0.45 \%$ during softer and harder states respectively.

On 10/05/2011, the rate is steady at around 170 counts/s as in the $\chi_{3}$ class. The object is in a hard state. A QPO at around $5 \mathrm{~Hz}$ is observed during this observation. Here the amount of $\mathrm{CE}$ is around $0.8 \%$. CE remains more of less steady for the entire observation dwell.

On 20/05/2011, the rate is steady at around 170 counts/s and the light curve resembles that of the $\chi_{2}$ class of GRS $1915+105$. The object is in a hard state. A QPO at around $4 \mathrm{~Hz}$ is observed. Here CE remains steady around $0.4 \%$ throughout the entire observation dwell.

On $17 / 07 / 2011$, the rates vary in the range of $50-200$ counts/s. This is a variable intermediate class, most likely a part of the $\beta$ type class of GRS $1915+105$, and no fixed periodicity is observed during this observation. Here, CE is varying from $0.04 \%$ to $0.4 \%$ during its softer and harder states respectively.

On $29 / 07 / 2011$, the rate is around 300 counts/s. During this observation no quasi periodic oscillation (QPO) is observed. Average CE is $\sim 0.08 \%$. This class looks very similar to the $\mu$ class of GRS $1915+105$.

On $31 / 07 / 2011$, the rate changes to around $150-350$ counts/s. This light curve looks similar to $\nu$ type variability class of GRS $1915+105$. Here the flickering takes place around each $10 \mathrm{sec}$ interval. Average CE varies around $\sim 0.11 \%$. No distinct QPO is observed during this class.

On $02 / 08 / 2011$, the rate remains in the range of $\sim 100-200$ counts/s. The light curve shows a similar variability as in the $\kappa$ class of GRS $1915+105$. Some periodicity of $\sim 10-15 \mathrm{sec}$ is observed also. The average CE is around $\sim 0.16 \%$ during this observation.

On 04/08/2011, the rate varies in the range of 200 to 350 counts/s. The light curve is variable as in $\rho$ class of GRS $1915+105$. The interval of each 'heartbeat' is around $25 \mathrm{sec}$. During this observation, CE is varying around $0.07 \%-0.2 \%$ during their softer and harder states respectively. We plot CE variation for the 04/08/2011 observation of IGR 17091-3624 data as in Fig. 3(a). 

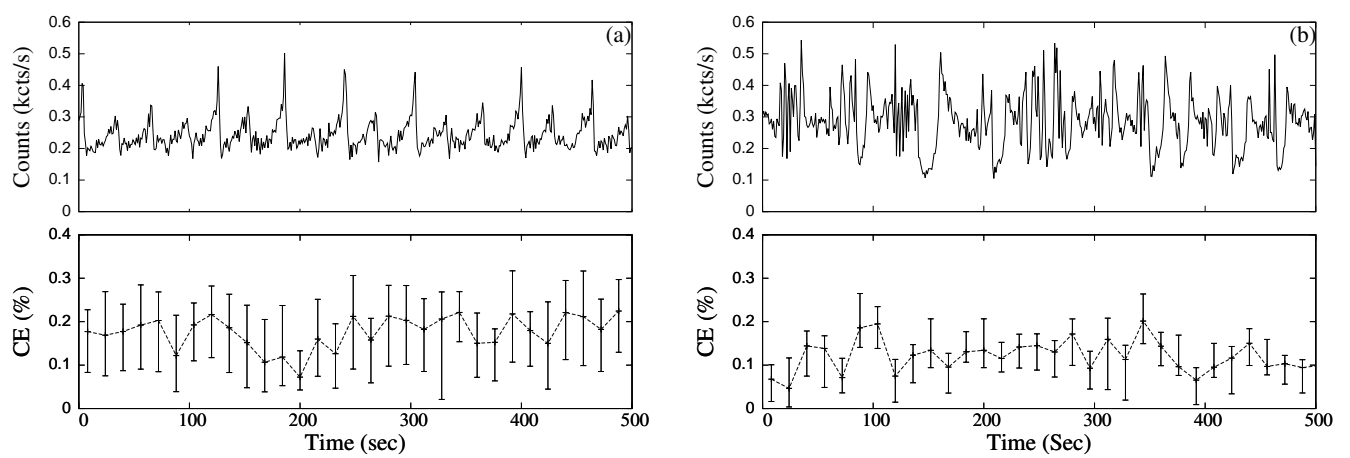

Figure 3: (a) Top panel shows 2.0 - $40 \mathrm{keV}$ and 1.0 sec time bin RXTE-PCA light curve of IGR 17091-3624 of 04/08/2011 observation. Bottom panel shows variation of average CE with time as obtained from $16 \mathrm{~s}$ binned data for this observation. (b) Top panel shows 2.0 - $40 \mathrm{keV}$ and $1.0 \mathrm{sec}$ time bin RXTE-PCA light curve of IGR 17091-3624 of 24/09/2011 observation. Bottom panel shows variation of average $\mathrm{CE}$ with time as obtained from $16 \mathrm{~s}$ binned data for this observation.

In Fig. 3(a), we plot CE as a function of time. Since the spectra are necessarily from a $16 \mathrm{~s}$ bin, CE does not show the same rise and fall as that of the light curve because of averaging out effects arising out of the minimum bin time constraints.

On 24/09/2011, the rate varies between $100-500$ counts/s. The light curve shows a variable shape but it does not show any regular periodicity. This can be treated to be a variable intermediate class, likely to be a part of $\lambda$ class of GRS 1915+105. During this observation, CE is found to vary between $0.04 \%$ and $0.2 \%$ in the burst-off and burst-on states respectively. We plot CE variation for this observation of IGR 17091-3624 data as in Fig. 3(b). In Fig. 3(b), we plot CE as a function of time. Since the spectra are necessarily from a $16 \mathrm{~s}$ bin, CE does not show the same rise and fall as that of the light curve because of averaging out effects arising out of the minimum bin time constraints.

On $22 / 10 / 2011$, the rate is around 140 counts/s. In this observation also, no clear periodicity is observed. On an average, $\mathrm{CE}$ is $\sim 0.10 \%$ similar to that in the $\mu$ class of GRS $1915+105$.

Finally, on 26/10/2011, the rate varied between $300-350$ counts/s. This variable class also looks like the $\mu$ class of GRS $1915+105$ with no clear periodicity. In this observation, $\mathrm{CE}$ varies in the range of $0.04 \%$ to $0.1 \%$. 


\section{Discussion \& Conclusion}

We compute CE for each observation. Note that in no occasion, transition from one variability class to another was observed during a single observation dwell. In future, with continuous monitoring such transitions are expected to be seen.

In order to show the mass independent aspect of $\mathrm{CE}$, we first show that conventional photon ratios do not look similar even when light curves of the same variability class of the objects are chosen. In Fig. 4(a), we show the 2 - $40 \mathrm{keV}$ data of 04/08/2011 PCA observation of IGR 17091-3624 which look similar to the so-called $\rho$ class data of GRS 1915+105 in Fig. 4(b). In Fig. 4(c), we draw the color-color diagram of GRS 1915+105 (Fig. 4b) following Belloni et al. (2000) with $2-5 \mathrm{keV}, 5-13 \mathrm{keV}$, and $13-60 \mathrm{keV}$ energy bands with black crosses along with gray error bars. When we repeat the exercise for IGR 17091-3624 (Fig. 4a) in Fig. 4(c) with black stars and gray error bars, the ratio looks different, more like that of the $\phi$ class of (Belloni et al., 2000). With suitable adjustment of energy ranges one can try to make them look similar, but this exercise is difficult to justify. What is clear is that soft and hard photons with preassigned energy bands are not useful in classifying variabilities. We need to take resort to a quantity which is independent of the mass of the black hole.

In Fig. 5, we plot average CE in each class for IGR 17091-3624 with filled squares. Average CEs are placed in increasing order. Dates of observations are given on the upper X-axis (see, Table. 1). For the sake of comparison, we plot average CEs of GRS 1915+105 with hollow triangles for the observed variability classes reported in Chakrabarti et al. (2005); Pal et al. (2011, 2013). Two sets of CEs of GRS 1915+105 (triangles) are taken from Fig. 4 of Pal et al. (2013). Shaded boxes group together light curves of 'similar appearance' from IGR 17091-3624 and GRS 1915+105 which were either reported by others or were discovered by us (see, Fig. 1). Each average CE (filled square or triangle) has an 'error bar' representing excursion of CE. within that class. The upper limits come from the highest value of positive $90 \%$ confidence error-bars and the lower limits come from the highest value of negative 90\% confidence error-bars. See, Pal et al. (2013) for details.

In Fig. 1, we observe that the objects exhibit variabilities of similar appearance but the time scales are vastly different. For $\nu, \rho$ or $\kappa$ type data, time-scale to complete an oscillation in IGR 17091-3624 is much shorter than that in GRS 1915+105. As Chakrabarti \& Manickam (2000) pointed out, 

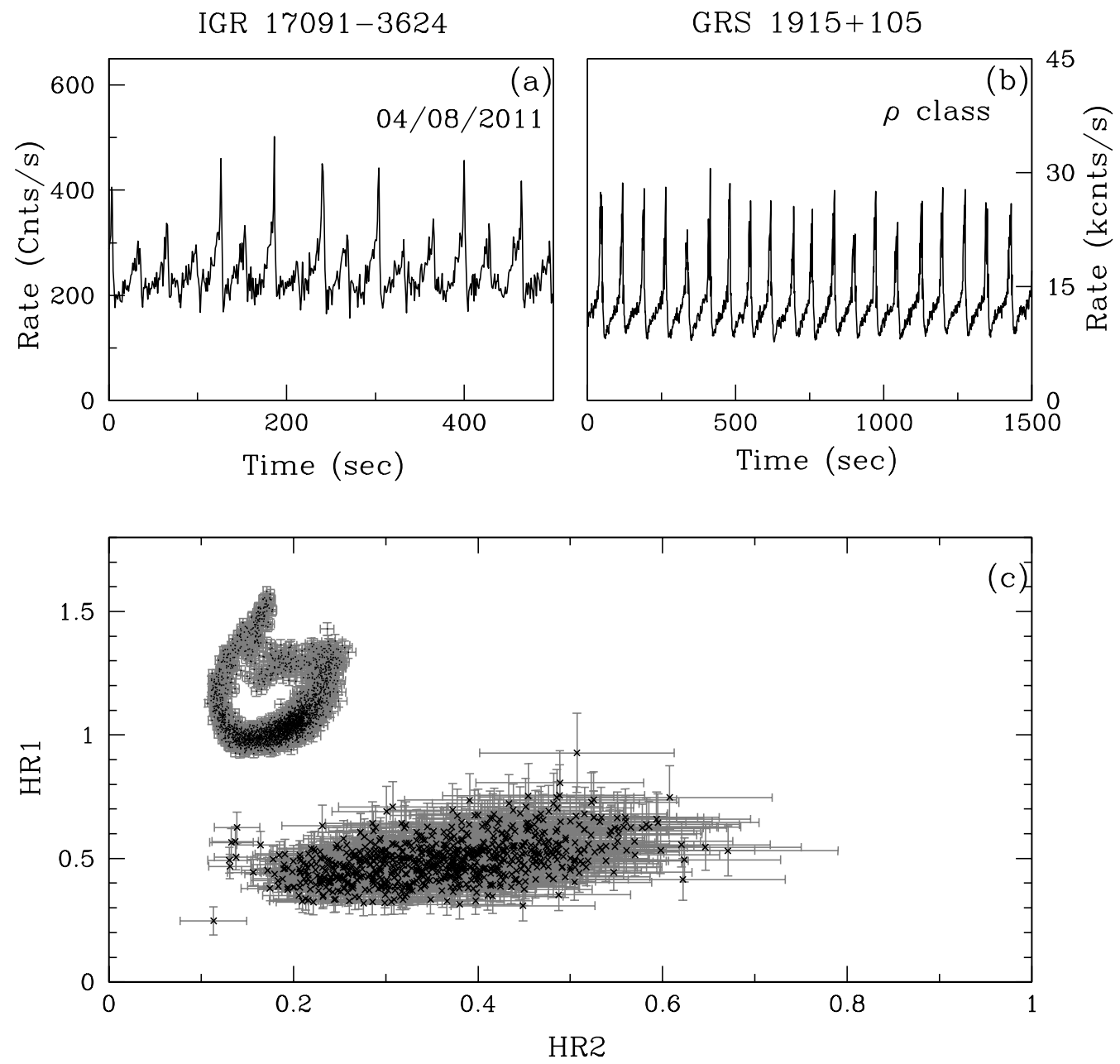

Figure 4: (a) 2 - $40 \mathrm{keV}$ and $1 \mathrm{sec}$ time bin RXTE-PCA light curve of IGR 17091-3624 on 04/08/2011. (b) $2-40 \mathrm{keV}$ and $1 \mathrm{sec}$ time bin RXTE-PCA light curve of $\rho$ class of GRS 1915+105. Following Belloni et al. (2000) with 2-5 keV, 5-13 keV and 13-60 keV energy bands we draw (c) Color-color diagram of (a) in black cross with gray error-bars, color-color diagram of (b) in black stars with gray error-bars. They look totally different. This indicates that the notion of soft and hard photons may be totally different in these two objects implying that the masses should be different. 


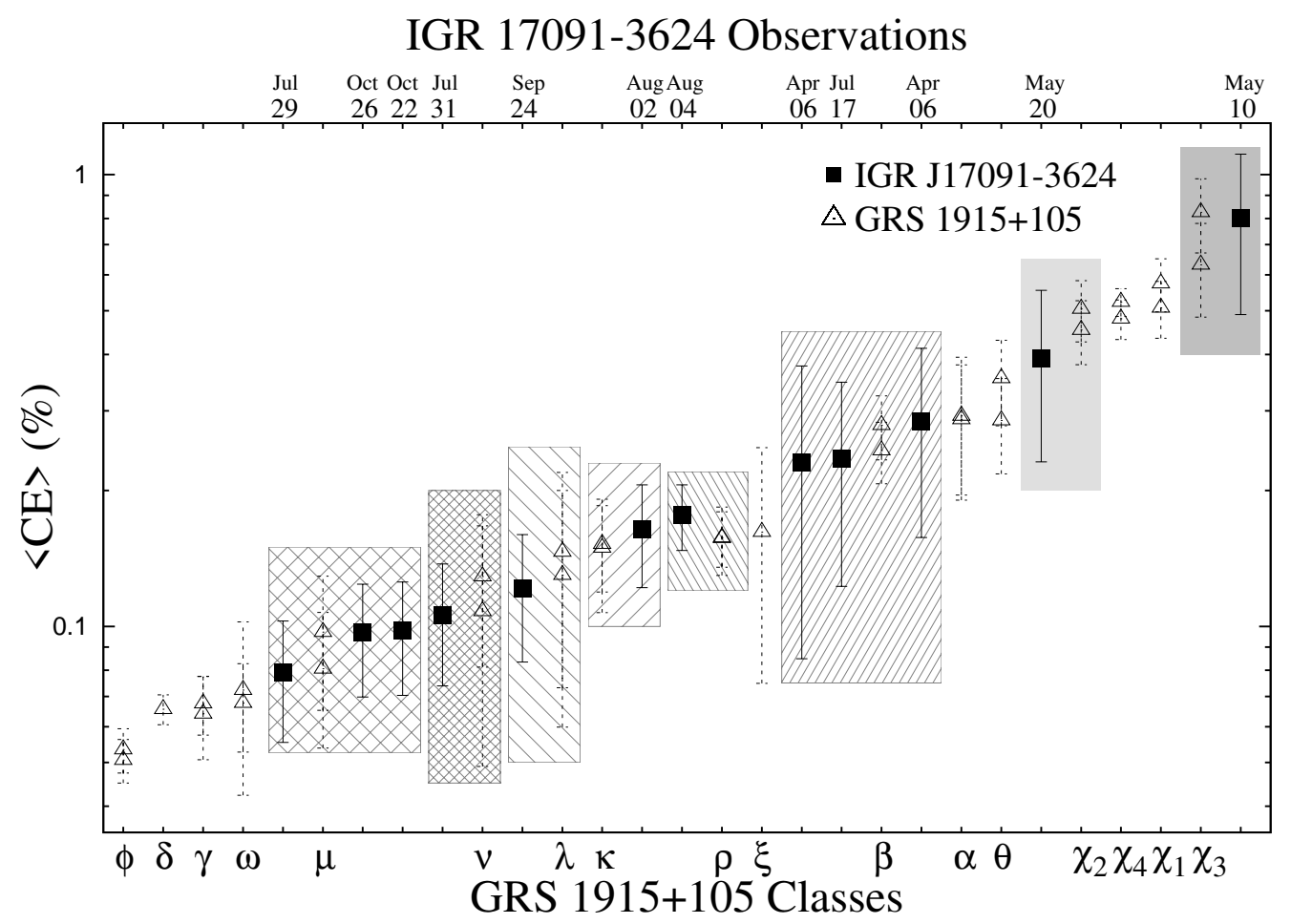

Figure 5: Average CE of IGR 17091-2634 (filled square) for the 2011 outburst drawn in ascending order is compared with average CE of GRS 1915+105 (hollow triangles). CE values of GRS 1915+105 (taken from the Fig. 4 of Pal et al. (2013)). 'Error bars' represent amount of excursion of 16 s averaged CEs (including their $90 \%$ confidence level error-bars) within these variability classes. Shaded boxes contain CE of GRS 1915+105 (two sets) and IGR 17091-3624 for which the light curves superficially are similar. Clearly, light curves of similar appearances produce roughly similar CEs. 
the alternate softer and harder spectra in these intermediate states could be due to the interaction between the jets and the disk matter where the base of the jet (the post-shock region) changes its optical depth periodically. This generally points to the fact that the black hole in IGR 17091-3624 could have much smaller mass than that in GRS 1915+105. However, Fig. 5 shows that CEs of the two black hole candidates are very close to each other in all the common variability classes. This shows that perhaps the number of hard and soft photons also scaled in the same way as the mass of the black holes, but their ratio remains the same. We have another reason to believe that the masses of IGR 17091-3624 and GRS 1915+105 are different. In GRS $1915+105$, the variability classes were distinguished on the basis of colorcolor diagrams. However, in Fig. 4c, we demonstrated that these diagrams drawn using fixed energy bands could be misleading. They look completely different even when the light curves are similar. This means that the so-called soft and hard photons do not belong to the same energy bands in these two objects. This can be generally possible only if the masses are different. Note that this conclusion is solely based on spectral and timing features and the distances of these objects are immaterial. There is no possibility that the accretion rates of the disk and the halo can conspire to reproduce the same spectral features at different timescales consistently for so many variability classes. However, even then, CE values that we compute, are found to have similar values for both the objects for the same class. Thus we believe that the evolution of the Comptonizing cloud in presence of seed photons of the standard disk, takes place exactly the same way in both the objects.

From our study we make some more observations:

(a) Several classes of light curves are not yet detected in IGR 17091-3624 (e.g., softer variability classes such as $\phi, \delta, \gamma$ and $\omega$ ). (b) No variability class transition has been observed within a single dwell (unlike in GRS 1915+105 where this was seen in several IXAE observations. See, Chakrabarti et al. (2005)). We predict that given sufficient observation time, IGR 17091-3624 would also have similar CE values in yet unobserved variability classes and the same sequence in class transitions as that of GRS $1915+105$ will prevail. On the other hand, given that the accretion rate in IGR 17091-3624 is perhaps much lower (Altamirano et al., 2011c), we suspect that it may not show the softer variability classes.

Near equality of average CE in IGR 17091-3624 and GRS 1915+105 leads us to believe that average $\mathrm{CE}$ may characterize variability classes uniquely for any stellar mass black holes. The scaling behavior of the Compton cloud 
and the standard disk only establishes that the Compton cloud is an integral component of the accretion flow itself as in the two-component advection flow solution of CT95. The conventional color-color diagrams with fixed energy bands cannot uniquely characterize the classes, since the physical origin of photons of a given energy band is different when the mass changes. This is not to say that the conventional color-color diagrams are not useful. Classifications of spectral states of neutron stars are successfully made on the basis of color-color diagrams from their energetic radiations (Hasinger, 1987; Hasinger \& van der Klis, 1989; Schulz et al., 1989). Since the masses

of neutron stars fall within a narrow range, these diagrams drawn using fixed sets of energy bands, can be used to classify all the neutron stars easily. However, stellar mass black holes are known to have masses in the range of $3-20 M_{\odot}$ (Remillard \& McClintock, 2006) and this creates an obvious problem.

Massive and super-massive black holes do have spectral states similar to stellar mass black holes. Indeed, the two-component advective flow model was first postulated for the active galaxies due to easy availability of low angular momentum matter in such systems Chakrabarti (1995). They also show short and long term variabilities (e.g., Cameron et al. (2012); Emmanoulopoulos et al. (2013) and references therein). It is not unlikely that the CE defined in our method will have similar values in these objects as well. This interesting aspect will be explored separately elsewhere.

\section{Acknowledgment}

PSP Acknowledges SNBNCBS-PDRA Fellowship.

\section{References}

Altamirano, D., Belloni, T. \& Krimm, H. et al., IGR J17091-3624 undergoes 'heartbeat' oscillations similar to those of GRS 1915+105, ATel, 3230, 1-1, 2011a.

Altamirano, D., Belloni, T. \& Krimm, H. et al., RXTE observations strengthen the similarities between the black hole candidates IGR J170913624 and GRS 1915+105, ATel, 3299, 1-1, 2011b.

Altamirano, D., Belloni, T. \& Linares, M. et al., The Faint "Heartbeats" of IGR J17091-3624: An Exceptional Black Hole Candidate, APJ, 742, 17-23, 2011c. 
Altamirano, D., Linares, M. \& van der Klis, M. et al., Discovery of $10 \mathrm{mHz}$ quasi-periodic oscillations likely from IGR J17091-3624, ATel, 3225, 1-1, 2011d.

Altamirano, D. \& Belloni, T., Discovery of High-frequency Quasi-periodic Oscillations in the Black Hole Candidate IGR J17091-3624, APJ, 747, 4-9, 2012.

Belloni, T., Klein-Wolt, M. \& Mendez, M. et al., A model-independent analysis of the variability of GRS 1915+105, A\&A, 355, 271-290, 2000.

Chakrabarti, S.K., Accretion Disks in Active Galaxies: The Sub-Keplerian Paradigm, NYASA, 759, 546-549, 1995.

Chakrabarti, S. K. \& Titarchuk, L. G., Spectral Properties of Accretion Disks around Galactic and Extragalactic Black Holes, APJ, 455, 623-639, 1995

Chakrabarti, S. K. \& Manickam, S. G., Correlation among Quasi-Periodic Oscillation Frequencies and Quiescent-State Duration in Black Hole Candidate GRS 1915+105, APJ, 531, 41-44, 2000.

Chakrabarti, S. K., Nandi, A. \& Chatterjee, A. K. et al., Class transitions and two component accretion flow in GRS 1915+105, A\&A, 431, 825-830, 2005.

Chaty, S., Rahoui, F. \& Foellmi, C. et al., Multi-wavelength observations of Galactic hard X-ray sources discovered by INTEGRAL. I. The nature of the companion star, A\&A, 484, 783-800, 2008.

Corbel, S., Rodriguez, J. \& Tzioumis, T. et al., Radio emission from IGR J17091-3624 observed with the ATCA, ATel, 3167, 1-1, 2011.

Cameron, D. T., McHardy, I. \& Dwelly, T et al., Correlated Xray/ultraviolet/optical variability in the very low mass AGN NGC 4395, MNRAS, 422, 902-912, 2012.

Emmanoulopoulos, D., Papadakis, I. E. \& Nicastro, F. et al., X-ray spectral analysis of the low-luminosity active galactic nucleus NGC 7213 using long XMM-Newton observations, MNRAS, 429, 3439-3448, 2013.

Hasinger, G., A classification of fast quasi-periodic X-ray oscillators - Is 6 HZ a fundamental frequency? A\&A, 186, 153-158, 1987. 
Hasinger, G.; van der Klis, M., Two patterns of correlated X-ray timing and spectral behaviour in low-mass X-ray binaries, A\&A, 225, 79-96, 1989.

Kennea, J. A. \& Capitanio, F., Swift/XRT Observations of IGR J17091-3624 and IGR J17098-3628, ATel, 1140, 1-1, 2007.

Krimm, H. A., Barthelmy, S. D. \& Baumgartner, W et al., Swift/BAT reports renewed activity from IGR J17091-3624, ATel, 3144, 1-1, 2011.

Krimm, H. A. \& Kennea, J. A., Swift/XRT Observations Confirm that IGR J17091-3624 is in Outburst, ATel, 3148, 1-1, 2011.

Kuulkers, E., Lutovinov, A. \& Parmar, A. et al. Igr J17091-3624, ATel, 149, $1-1,2003$.

Makishima, K., Maejima, Y. \& Mitsuda, K. et al., Simultaneous X-ray and optical observations of GX 339-4 in an X-ray high state, APJ, 308, 635-643, 1986.

Muno, M. P., Morgan, E. H. \& Remillard, R. A., Quasi-periodic Oscillations and Spectral States in GRS 1915+105, APJ, 527, 321-340, 1999.

Pahari, M., Bhattacharyya, S. \& Yadav, J. S. et al., Evidence of two unique variability classes from IGR J17091-3624, MNRAS, 422, 87-90, 2012.

Pal, P. S., Chakrabarti, S. K. \& Nandi, A., Evidence of Variation of the Accretion Flow Geometry in GRS 1915+105 from IXAE and RXTE Data, IJMPD, 20, 2281-2289, 2011.

Pal, P. S., Chakrabarti, S. K. \& Nandi, A., Comptonization efficiencies of the variability classes of GRS 1915+105, AdSpR, 52, 740-759, 2013.

Pal, P. S. \& Chakrabarti, S. K., A study of the variation of geometry of accretion flows of compact objects through timing and spectral analysis of their outbursts, MNRAS, 440, 672-682, 2014.

Paul, B. Agrawal, P. C. \& Rao, A. R. et al., Rapid X-ray variability of the superluminal source GRS 1915+105, A\&A, 320, 37-40, 1997.

Paul, B., Agrawal, P. C. \& Rao, A. R. et al., Quasi-regular X-Ray Bursts from GRS 1915+105 Observed with the IXAE: Possible Evidence for Matter Disappearing into the Event Horizon of the Black Hole, ApJ, 492, 63-66, 1998. 
Remillard, R. A. \& McClintock, J. E., X-Ray Properties of Black-Hole Binaries, ARA\&A, 44, 49-92, 2006.

Rodriguez, J., Corbel, S. \& Caballero, I. et al. First simultaneous multiwavelength observations of the black hole candidate IGR J17091-3624. ATCA, INTEGRAL, Swift, and RXTE views of the 2011 outburst, A\&A, 533L, 4-8, 2011.

Schulz, N. S., Hasinger, G. \& Truemper, J., Spectral classification of low-mass X-ray binary (LMXB) energy spectra with color-color diagrams, A\&A, 225, 48-68, 1989.

Sobczak, G. J., McClintock, J. E. \& Remillard, R. A. et al., RXTE Spectral Observations of the 1996-1997 Outburst of the Microquasar GRO J165540, APJ, 520, 776-787, 1999.

Titarchuk, L. G., Generalized Comptonization models and application to the recent high-energy observations, APJ, 434, 570-586, 1994.

Torres, M. A. P., Jonker, P. G. \& Steeghs, D. et al., The optical and nearinfrared counterpart to IGR J17091-3624, ATel, 3150, 1-1, 2011.

Yadav, J. S., Rao, A. R. \& Agrawal, P. C. et al., Different Types of X-Ray Bursts from GRS 1915+105 and Their Origin, ApJ, 517, 935-950, 1999. 